Article

\title{
Identifying Quark Matter in Hybrid Stars through Relativistic Tidal Deformations
}

\author{
Bryen Irving ${ }^{1,2}$, Thomas Klähn ${ }^{1, *}$, Prashanth Jaikumar ${ }^{1}$, Marc Salinas ${ }^{1}$ and Wei Wei ${ }^{3}$ \\ 1 Department of Physics \& Astronomy, California State University Long Beach, Long Beach, CA 90840, USA \\ 2 Department of Physics, Stanford University, Stanford, CA 94305, USA \\ 3 College of Science, Huazhong Agricultural University, Wuhan 430070, China \\ * Correspondence: thomas.klaehn@csulb.edu
}

Received: 9 May 2019; Accepted: 23 August 2019; Published: 30 August 2019

\begin{abstract}
We study a specific model of neutron star matter that supports a phase transition to quark matter at high density and examine parameter ranges for consistency with the mass-weighted tidal deformability of $\tilde{\Lambda}=300_{-230}^{+420}$ for a mass ratio of $q \in[0.73,1.0]$, as inferred from observations of gravitational waves from the binary neutron star merger event GW170817. By using this observation to restrict the parameter space for the equation of state (EoS) model used throughout this study, we aim to assess the possibility of a potential solution to the masquerade and flavor camouflage problems for hybrid EoS models. Assuming the two stars have the same EoS, in which the Dirac-Brueckner-Hartree Fock (DBHF) nuclear model transitions to the vBag quark model, we see if the parameter space of these hybrid model stars are restricted due to the adherence to the reported $\Lambda_{1.4} \in[70,580]$ and $M_{\max } \in[2.01,2.16] M_{\odot}$ constraints. Upon completion, we find that, while the parameter space for our model does get restricted, it does not ultimately resolve the masquerade and flavor camouflage problems.
\end{abstract}

Keywords: compact stars; mixed phases; gravitational waves; equation of state; tidal deformability; QCD; quark-hadron phase transitions

\section{Introduction}

Understanding the equation of state of strongly interacting matter under the extreme conditions in compact stars is a key problem of interest in astrophysics [1,2]. One of the most pressing issues revolves around understanding models that experience a phase transition from hadronic to deconfined quark matter at densities several times that of nuclear saturation. While modern observational techniques have allowed for constraints to the mass-radius relations of these stars to be generated, the so-called "masquerade" [3] and "flavor camouflage" [4] problems still persist. As it stands, based on current observational techniques, a hybrid star with quark matter in its interior is indistinguishable from that of an ordinary Neutron Star (NS) - even more so if mixed phases exist. Furthermore, when considering phase transitions from nuclear to two-flavor (2f) or three-flavor (3f), matter signatures in the mass-radius relations become effectively camouflaged when compared to their purely nuclear counterparts. Therefore, it becomes apparent that additional methods are needed to further understand these hybrid models.

On 17 August 2017, the Advanced LIGO/VIRGO scientific collaborations intercepted signals of gravitational radiation [5] —in addition to - the accompanying electromagnetic signal [6,7] from coalescing neutron stars (NSs) - thus ushering in a new age of multi-messenger astronomy. While the GW170817 signal has provided a long awaited tool needed to probe the interior of these bodies, speculation about its 
ability to resolve various properties of hybrid configurations has arisen [8-10]. It has been shown [11-18] that, during the inspiral phase of binary coalescence, each star induces a static tidal field upon one another. These tidal fields then generate deformations in the structure of each star, consequently draining energy from the orbit, and expediting coalescence. These tidal effects are encoded into the gravitational wave (GW) signatures and, by connecting the geometric structure and interior properties, the nature of dense matter within the star's interior can potentially be decoded.

This study aims to illustrate a framework to understand how the tidal interactions from coalescing neutron stars can be used to potentially identify quark matter in hybrid configurations. In Section 2, we demonstrate how tidal deformations arise through the lens of modern gravitational theory. Section 3 provides a brief overview of the nuclear and quark matter models used for this study, while defining the necessary conditions for a phase transition to occur. Section 4 lists the constraints we use and our results and concluding remarks are gathered in Section 5. (Unless otherwise specified: $G=c=\hbar=1$ ).

\section{Relativistic Tidal Deformations}

\subsection{Gravitational Radiation}

In the context of gravitational radiation, the intertwined relation between the dynamics of a source's quadrupole moments and the surrounding external tidal field may be exploited. By using such relationships, new insights into the star's interior can be revealed from quantifiable parameters extracted from signals of gravitational radiation intercepted by ground based detectors [19]. To help with the extraction of theses parameters, match filtering techniques are typically applied to efficiently sift through signal noise and properly identify the waveform that corresponds with these parameters. Therefore, Post-Newtonian (PN) expansions of the system's signal and observables are taken to determine the dominant features. When assessing the source's tidal influence on the gravitational wave signal, the leading order contribution can be found in the gravitational wave phase in terms of the chirp mass $\mathcal{M}_{c}$, symmetric mass ratio $\eta$, and tidal deformability in the 5PN expansion term [12,20,21]:

$$
\delta \Psi_{\text {Tidal }}^{5 \mathrm{PN}}=-\frac{117}{256 \eta}\left(\frac{v}{c}\right)^{5} \tilde{\Lambda}=-\frac{117 \mathcal{M}_{c}^{5 / 3}}{256 \eta^{2}} \frac{(G \pi)^{5 / 3}}{c^{5}} f^{5 / 3} \tilde{\Lambda}
$$

Recalling Kepler's $3^{r d}$, the phase contribution $\delta \Psi_{\text {Tidal }}^{5 \mathrm{PN}}$ is conveniently expressed in terms of the gravitational wave frequency $f$ as opposed to its velocity $v$. The mass-weighted average, or effective, tidal deformability $\widetilde{\Lambda}$ serves as a parameter to model the dominant tidal behavior from each star in the binary system

$$
\tilde{\Lambda} \equiv \frac{16}{13} \frac{(1+12 q) \Lambda_{1}+(q+12) q^{4} \Lambda_{2}}{(1+q)^{5}} \quad \text { where } \quad q=\frac{M_{2}}{M_{1}} \cdot \leq 1
$$

Here, $\widetilde{\Lambda}$ is expressed in terms of the respective masses $M_{1}, M_{2}$ and tidal deformability $\Lambda_{1}, \Lambda_{2}$ from each star ${ }^{1}$. The difficulty in extracting information about an individual star has been well established, so in the same vein in applying the more constrained chirp mass to understand the mass contributions, the effective tidal deformability provides a more efficient tool to decode the tidal behavior within a binary system.

1 The factor of $16 / 13$ serves as a normalization factor, such that for $q=1, \widetilde{\Lambda}=\left(\Lambda_{1}+\Lambda_{2}\right) / 2$. 


\subsection{Modeling Tidal Deformations}

Since $\Lambda$ quantifies the extent of deformations, we expect to see for a given tidal field that it is important that we properly find a correlation between the geometric and matter properties of our desired system. Solving the field equations for the equilibrium structure $\bar{G}_{\mu v}=8 \pi \bar{T}_{\mu v}$ yield the Tolman-Oppenheimer-Volkoff (TOV) Equations [22,23]

$$
\begin{aligned}
\frac{d p(r)}{d r} & =-\frac{[\epsilon(r)+p(r)]\left[m(r)+4 \pi r^{3} p(r)\right]}{r[r-2 m(r)]}, \\
\frac{d m(r)}{d r} & =4 \pi r^{2} \epsilon(r),
\end{aligned}
$$

where $m$ is the mass at some radial distance $r$, and $p, \epsilon$ are the pressure and energy density of the comprising fluid in the star. Provided an equation of state (EoS) model, where a relation between the pressure and energy density is established, the equations above can be integrated concurrently from the star's core until the pressure and energy density become approximately zero at the surface-resulting in the mass and radius of the star. Solving for the linear perturbation term $\delta G_{\mu \nu}^{(1)}=8 \pi \delta T_{\mu \nu}^{(1)}$ produces a relation involving the perturbation potential $H_{\ell}(r)[14,18]$ :

$$
r^{2} H_{\ell}^{\prime \prime}(r)+A(r) r H_{\ell}^{\prime}(r)-B_{\ell}(r) H_{\ell}(r)=0,
$$

where in the interior of the star:

$$
\begin{aligned}
A(r) & =2 \bar{g}_{r r}\left[1-\frac{3 m}{r}-2 \pi r^{2}(\epsilon+3 p)\right], \\
B_{\ell}(r) & =\bar{g}_{r r}\left[\ell(\ell+1)-4 \pi r^{2}(\epsilon+3 p)\left(3+c_{s}^{-2}\right)\right] .
\end{aligned}
$$

Here, $c_{s}$ is the speed of sound in the medium, $\ell$ is the multipole order, and $\bar{g}_{r r}$ is the r-r component of the Schwarzschild metric.

For the purpose of computation, in the limit as the radius approaches the center of the star (i.e., $r \rightarrow 0$ ), the perturbation potential behaves $\lim _{r \rightarrow 0} H_{\ell} \sim r^{\ell}$. To avoid computations diverging towards the center of the star, it is standard to convert Equation (5) into a logarithmic form like so:

$$
r y_{\ell}^{\text {int' }}(r)+y_{\ell}^{\text {int }}(r)\left[y_{\ell}^{\text {int }}(r)-1\right]+A(r) y_{\ell}^{\text {int }}(r)-B_{\ell}(r)=0 \quad \text { where } \quad y_{\ell}^{\text {int }}=\frac{r H_{\ell}^{\prime}(r)}{H_{\ell}(r)} .
$$

The interior solution can be solved in tandem with the TOV Equations and has an initial condition of $y_{\ell}^{\text {int }}(0)=\ell$ due to the $r \rightarrow 0$ limit for $H_{\ell}$.

In the exterior region, the potential region reduces to function dependent on the associated Legendre Polynomials of the 1st and 2nd kind:

$$
H_{\ell}^{\mathrm{ext}}=a_{\ell}^{Q} \hat{Q}_{\ell 2}(x)+a_{\ell}^{P} \hat{P}_{\ell 2}(x) \quad \text { for } \quad x=\frac{r}{M}-1 .
$$

The constants $a_{\ell}^{Q}, a_{\ell}^{P}$ serves as a normalization factor such that, in the far limit $r \rightarrow \infty$, the "growing" contribution $\hat{P}_{\ell 2}(x) \sim x^{\ell}$ is equal to the "shrinking" contribution $\hat{Q}_{\ell 2}(x) \sim x^{-(\ell+1)}$. To create a matching 
condition at the surface, the logarithmic form [13] expressed by Equation (8) is as follows in the exterior region of the star:

$$
y_{\ell}^{\mathrm{ext}}(r)=\frac{r H_{\ell}^{\prime}(r)}{H_{\ell}(r)}=(1+x) \frac{\hat{P}_{\ell 2}^{\prime}(x)+a_{\ell} \hat{Q}_{\ell 2}^{\prime}(x)}{\hat{P}_{\ell 2}(x)+a_{\ell} \hat{Q}_{\ell 2}(x)}, \quad \text { where } \quad a_{\ell}=\frac{a_{\ell}^{Q}}{a_{\ell}^{P}} .
$$

At the surface of the star, the internal and external perturbation potentials are equal and can be summarized by $y_{\ell}^{\text {int }}(R)=y_{\ell}^{\text {ext }}(R)=Y_{\ell}$. Matching the internal and external gravitational potentials produces the following definition of the polar tidal Love number [24] in terms of the tidal deformability and compactness parameter $\mathcal{C}=M / R$ :

$$
\lambda_{\ell}(2 \ell-1) ! ! R^{-(2 \ell+1)}=a_{\ell} \mathcal{C}^{2 \ell+1} \equiv 2 k_{\ell}^{\text {polar }} .
$$

For a given multipole order $\ell$, one can expect to detect dimensionless tidal deformability of the following form:

$$
\Lambda_{\ell}=\frac{\lambda_{\ell}}{M^{2 \ell+1}}=\frac{2 k_{\ell}^{\text {polar }}}{(2 \ell-1) ! ! \mathcal{C}^{2 \ell+1}}=\left.\frac{-1}{(2 \ell-1) ! !} \frac{\hat{P}_{\ell 2}^{\prime}(x)-\mathcal{C} Y_{\ell} \hat{P}_{\ell 2}(x)}{\hat{Q}_{\ell 2}^{\prime}(x)-\mathcal{C} Y_{\ell} \hat{Q}_{\ell 2}(x)}\right|_{x=\mathcal{C}^{-1}-1}
$$

Since gravitational waves are produced by accelerating quadrupole moments $(\ell=2)$, the tidal deformability that one can expect to detect in ground-based detectors is of the following form:

$$
\begin{aligned}
\Lambda & =\frac{16}{15}[1-2 \mathcal{C}]^{2}[2-Y+2 \mathcal{C}(Y-1)] \\
& \times\left[\begin{array}{r}
2 \mathcal{C}[6-3 Y+3 \mathcal{C}(5 Y-8)]+4 \mathcal{C}^{3}\left[13-11 Y+\mathcal{C}(3 Y-2)+2 \mathcal{C}^{2}(1+Y)\right] \\
+3(1-2 \mathcal{C})^{2}[2-Y+2 \mathcal{C}(Y-1) \ln [1-2 \mathcal{C}]
\end{array}\right]^{-1},
\end{aligned}
$$

where $\Lambda=\Lambda_{\ell=2}$ and $Y=Y_{\ell=2}$. From the effective relation $\Lambda=f(M, R, E o S)$, we see how a star's shape and interior make up influence its behavior during the merger process. Typically from a given signal, a single value for the chirp mass and a range of potential component mass values are reported. Therefore, in order to assess the potential deformability of the various binary configurations, we can expect a range of values for $\Lambda_{1}, \Lambda_{2}$, and $\widetilde{\Lambda}$.

\section{Hybrid Equation of the State Model}

\subsection{Nuclear Matter}

When modeling the behavior of cold hadronic matter for astrophysical applications, it is common to approximate the energy per baryon using the parabolic expansion:

$$
E_{\text {nuc }}(n, x)=E(n, x=1 / 2)+S(n)(1-2 x)^{2},
$$

which is dependent on the baryonic density $n$ and the proton fraction $x=n_{p} / n$. Then, by applying simple thermodynamic relations

$$
\begin{gathered}
\epsilon_{\text {nuc }}(n, x)=n E_{\text {nuc }}(n, x), \\
P_{\text {nuc }}(n, x)=-\frac{\partial\left(\epsilon_{n u c} / n\right)}{\partial(1 / n)}=n^{2} \frac{\partial E_{\text {nuc }}}{\partial n}
\end{gathered}
$$


to an ensemble describing matter that adheres to the conditions of beta equilibrium and charge neutrality the nuclear pressure and energy density take the following form:

$$
\begin{aligned}
\epsilon_{\text {nuc }}(n, x) & =\sum_{j=n, p, e, \mu} \epsilon_{j}\left(n_{j}, x\right), \\
P_{\text {nuc }}(n, x) & =\sum_{j=n, p, e, \mu} P_{j}\left(n_{j}, x\right) .
\end{aligned}
$$

To efficiently parameterize the many-body behavior in the nuclear regimes, the Dirac-Brueckner-Hartree-Fock (DBHF) model [25-29] was chosen. This model has been well tested for neutron star applications [30] and is widely used throughout the field.

\subsection{Phase Transitions}

As one begins to approach high pressure regimes inside of a star, prior studies have suggested that a star's ingredient nuclear matter will begin to break down into its constituent parts-quarks. This phase transition softens the EoS, therefore reducing the tidal deformability. Therefore, the boundary conditions our model demand that a phase transition from nuclear to quark matter occur at the following circumstances $[4,31]$ :

$$
P_{\text {nuc }}\left(\mu_{B, \text { crit }}^{\text {nuc }}\right)=P_{\text {quark }}\left(\mu_{B, \text { crit }}^{\text {quark }}\right) .
$$

Here, the $P_{i}\left(\mu_{B, c r i t}^{i}\right)$ terms represent the pressure value at the critical baryon-chemical potential required for chiral transition. It is to be noted that the pressure differentials at $\mu_{B, \text { crit }}$ are different for each state of matter, and the behavior of these differentials is characteristic of different transitions. A Maxwell phase transition is signified by a discontinuous jump between states, whereas Gibbs transition results from a smooth transition due to mixed phases of matter between pure nuclear and pure quark regimes. To account for potential mixed phases of matter, the energy density for the entire model can be summed up by the following relation:

$$
\epsilon=(1-\eta) \epsilon_{n u c}+\eta \epsilon_{\text {quark }}
$$

with $\eta=V_{q} / V \in[0,1]$ defining the volume fraction of quark matter in our system. For a Maxwell transition, the volume fraction takes on discrete values of 0 or 1 depending on the regime of the star that is being assessed. In short, $\eta=0$ for the outer nuclear regions, and abruptly switches to $\eta=1$ for the inner quark matter regions. For a Gibbs transition, the energy density described by Equation (20) will have a fractional dependence on both terms due to the fractional value of $\eta$ inside of mixed phases.

To account for discontinuities due to a possible Maxwell transition, the in-medium speed of sound term in $B_{\ell}(r)$ can take a discontinuous form

$$
c_{s}^{-2}=\left.\frac{d \epsilon}{d p}\right|_{p \neq p_{d}}+\left(\epsilon_{+}-\epsilon_{-}\right) \delta\left(p-p_{d}\right)
$$

producing a smooth $c_{S}$ curve for the parabolic nuclear regimes that discontinuously jumps into a curve that is dependent on the behavior the quark matter located in the star's interior. Here, $p_{d}$ signifies the pressure located at the discontinuous jump, $\epsilon_{+}$the energy density approaching from the upper limit, and $\epsilon_{-}$the energy density approaching from the lower limit. Consequently, this forces $y_{\ell}^{\text {int }}$ to take the form of Equation (14) in [17]. It is to be noted that, since the speed of sound will be computed by numerical differentiation, it is important that the equation of state model is well-defined throughout all regimes of the star. This will result in a better representation of $c_{s}$, and thus a more accurate calculation for $\Lambda$. 


\subsection{Quark Matter}

When considering the behavior of quark matter, it is standard to build models accounting for non-interacting or interacting behavior, like the Bag and NJL models, respectively. The vector-interaction-enhanced (vBag) [32] model takes highlights from both schools of thought to effectively model quark matter phenomena in a linear fashion. For a generalized regime containing all three flavors of the light quarks, pressure and energy density as described by the vBag model are

$$
\begin{aligned}
& P_{\text {quark }}=\sum_{f=u, d, s} P_{v B a g, f}+B_{d c}, \\
& \epsilon_{\text {quark }}=\sum_{f=u, d, s} \epsilon_{v B a g, f}-B_{d c} .
\end{aligned}
$$

The Bag constant, $B_{d c}$, is a pressure term that regulates the chiral and deconfinement behavior of the system. Additionally, $P_{v B a g, f}$ and $\epsilon_{v B a g, f}$ are the pressure and energy density of a single quark flavor, respectively, and are as follows:

$$
\begin{aligned}
& P_{v B a g, f}\left(\mu_{f}\right)=P_{F G, f}\left(\mu_{f}^{*}\right)+\frac{K_{v}}{2} n_{F G, f}^{2}\left(\mu_{f}^{*}\right)-B_{\chi, f}, \\
& \epsilon_{v B a g, f}\left(\mu_{f}\right)=\epsilon_{F G, f}\left(\mu_{f}^{*}\right)+\frac{K_{v}}{2} n_{F G, f}^{2}\left(\mu_{f}^{*}\right)+B_{\chi, f},
\end{aligned}
$$

the $K_{v}$ parameter in the second term is a coupling constant that results from vector interactions, and $B_{\chi, f}$ is the Bag constant of a single flavor, The chemical potential, $\mu_{f}^{*}$, of the system is parameterized by the following relation:

$$
\mu_{f}=\mu_{f}^{*}+K_{v} n_{F G, f}\left(\mu_{f}^{*}\right) .
$$

In order to ensure that the phase transition from nuclear to quark occurs at the same chemical potential, we introduce the following effective Bag constant:

$$
B_{\text {eff }}=\sum_{f=u, d, s} B_{\chi, f}-B_{d c}
$$

The first term illustrates how $B_{\text {eff }}$ can be used in either case of quark flavor. In the case of two-flavor matter, we negate the Bag constant resulting from the strange quark contribution. For the hybrid models used in this study, two flavor configurations with $\left(K_{v}, B_{\text {eff }}\right)=(4,65),(4,70)$ and three flavor configurations with $\left(K_{v}, B_{\text {eff }}\right)=(17.5,95),(17,80)$ will be used-their resulting equation of state profiles can be seen in Figure 1 below. 

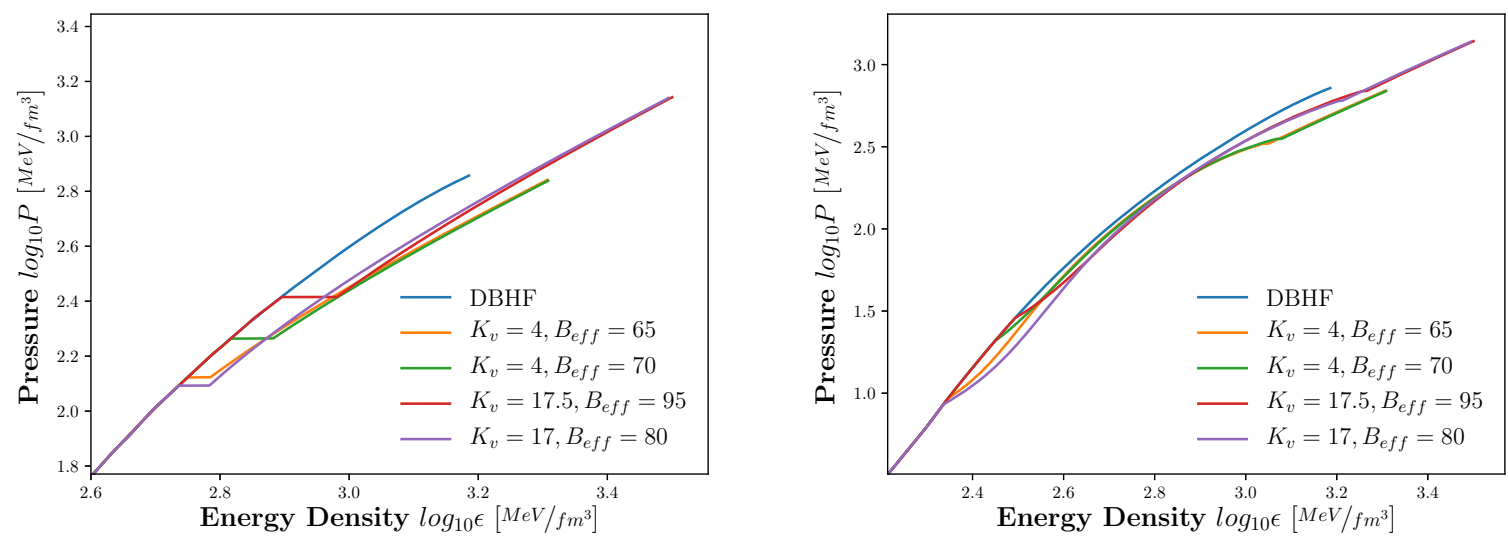

Figure 1. Equation of State Curves with Phase Transitions Nuclear Matter. The DBHF curve is the purely nuclear model, while the curves denoted with $K_{v}\left[\mathrm{GeV}^{-2}\right]$ and $B_{\text {eff }}\left[\mathrm{MeV} / \mathrm{fm}^{3}\right]$ values are the hybrid models that experience transitions from nuclear to quark matter. The image to the left displays hybrid configurations with a Maxwell construction, and the image to the right illustrates these same configurations with Gibbs configurations. While the images focus on the location of the phase transition in each respective model, these models continue to follow the same profile referenced in [30] down to pressure and density regimes typical of atmospheric regions.

\section{Constraints from the Tidal Contributions of Gravitational Radiation}

\subsection{GW170817 Results}

Current bounds for the effective tidal deformability-as reported by the LIGO-VIRGO collaboration [33] - has been placed at $\tilde{\Lambda}=300_{-230}^{+420}$ at a $90 \%$ confidence for low-spin priors. Furthermore, the deformability of a star at the Chandrasekhar mass has been reported at $\Lambda_{1.4}=\Lambda\left(1.4 M_{\odot}\right)=$ $190_{-120}^{+390}$ under the same conditions. At a $90 \%$ confidence level, the chirp mass was constrained to $\mathcal{M}_{c}=1.186 \pm 0.001$. Inferred component mass ranges were reported at $M_{1} \in[1.36,1.60] M_{\odot}$ and $M_{2} \in[1.16,1.36] M_{\odot}$ with a mass ratio $q \in(0.73,1.0]$ all at a $90 \%$ confidence level.

\subsection{Constraints}

Assuming that both stars in our system are comprised of the same EoS, we assess our models against the following tidal deformability constraints: (i) $\tilde{\Lambda} \in[70,720]$ for $q \in[0.73,1.0]$ (ii) $\Lambda_{1.4} \in[70,580]$. Furthermore, a host of prior studies [34,35] have suggested that a given EoS model should be able to comfortably sustain a maximum mass of approximately $M_{\max } \in[2.01,2.16] M_{\odot}$. It should be noted that recent parameter estimation studies that include the accompanying gamma-ray burst [6] and kilonova [7] signals have been conducted. Recent studies of the multi-messenger signals have placed different constraints on $\tilde{\Lambda}$, but, because hybrid EoS models were not taken into account in their study, these bounds may provide inconclusive results [36,37]. Therefore, for the purpose of this study, we will abide by the constraints reported in the LIGO report, and use the constraints from the multi-messenger analysis as a tentative requirement until models with hybrid EoS are developed. In short, we can begin to reduce the parameter space in our model by applying the above constraints. 


\section{Results}

\subsection{Mass-Radii Relation}

A common approach to understanding the type of star configurations produced by a given EoS model is to analyze the mass-radii relation of the set. For the purpose of this study, the hybrid models will be divided by the construction of its respective phase transition and assessed with respect to its purely nuclear counterpart. Looking at the mass-radius relation for the DBHF model in the Figure 2 below, it becomes apparent that it doesn't adhere to the maximum mass constraint as it is able to sustain stable configurations that exceed $2.16 M_{\odot}$. While the purely nuclear curve doesn't adhere to these constraints, it appears that a phase transition from nuclear to quark matter rectifies this issue. The parameters established in the vBag model control the type of curves that result after the transition from hadronic to quark matter occurs. The $K_{v}$ parameter controls the curve's stiffness, while the effective bag coefficient $B_{\text {eff }}$ triggers the location where the phase transition is along the nuclear curve. The aggressive phase transition associated with the Maxwell construction appears in the mass-radii relations as a sharp discontinuous transition to a quark matter branch. On the other hand, due to the appearance of mixed phases, hybrid models that experience a Gibbs transition produce mass-radius curves that have a smooth transition from the nuclear to quark matter branch.
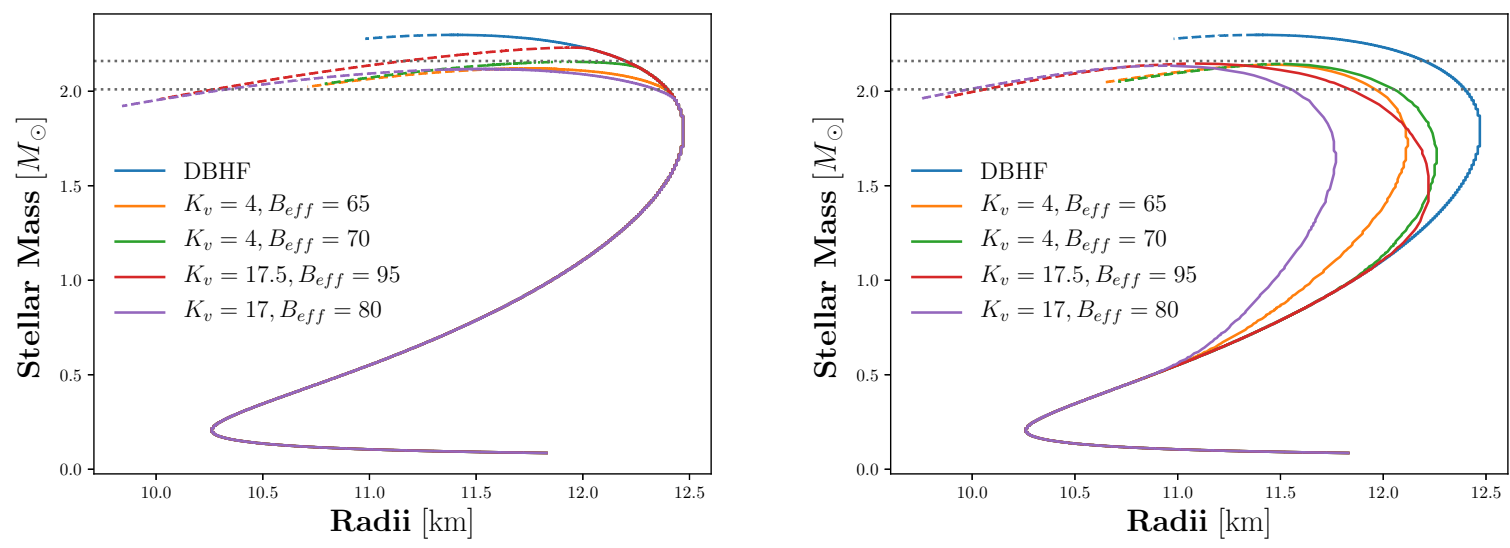

Figure 2. The mass-radius curves obtained solving the TOV Solution from equations (3) for the various hybrid configurations shown in Figure 1. The image to the left displays hybrid configurations with a Maxwell construction, and the image to the right illustrates these same configurations with Gibbs configurations. It is to be noted that the dashed portions of the Mass-Radii curves indicates regimes of unstable star configurations. The region bounded by the grey dashed lines represent the $M_{\max }$ constraints applied to our model.

Looking the hybrid models that underwent a Maxwell transition, in Figure 2, all but the $\left(K_{v}, B_{\text {eff }}\right)=$ $(17.5,95)$ models are able to sustain masses that meet the $M_{\max }$ requirements. Furthermore, it appears that the star configurations that occur after the transition for $\left(K_{v}, B_{\text {eff }}\right)=(17.5,95)$ are all unstable, while the others contain both stable and unstable configurations. For the models that undergo Gibbs transitions, all are able to meet the $M_{\max }$ constraints. Additionally, these curves illustrate that the maximum radius for star produced under these models is slightly less than $12.5 \mathrm{~km}$.

\subsection{Tidal Relations}

Moving to the tidal relations, all of the models—including the nuclear model-adhere to the effective tidal deformability constraint. 
Further analysis of the values reported in Table 1 shows that the effective tidal deformability is fairly stiff across the mass-ratio spectrum reported by LIGO. While a recent study [36] showed that this held for various nuclear models, these results show that this relation for $\tilde{\Lambda}(q)$ can also hold for hybrid models as well. With the nominal value for $\tilde{\Lambda}$ being reported at 300 , it appears that the $\left(K_{v}, B_{\text {eff }}\right)=(17,80)$ Gibbs-constructed hybrid model is more favored by this constraint. The resulting $\Lambda_{1.4}$ values presented in Table 2 show that the Maxwell-constructed models used in our study are not feasible as they all exceed the upper bound of the $\Lambda_{1.4}$ constraint. Additionally, with a nominal value of 190 being reported for $\Lambda$, the $\left(K_{v}, B_{\text {eff }}\right)=(17,80)$ Gibbs-constructed hybrid model is also more favored by this tidal constraint too.

Table 1. The effective tidal deformability $\tilde{\Lambda}$ for a given hybrid equation of state model over the mass-ratio range $q \in[0.73,1.00]$. The parameter pairings in the leftmost column denote the vBag parameters for the quark model that the DBHF model transitions into. For reference, the purely nuclear DBHF model yields values of $\tilde{\Lambda} \in[663,690]$. The effective tidal deformability $\tilde{\Lambda}$ and mass ratio $q$ are dimensionless.

\begin{tabular}{ccc}
\hline & Maxwell Construction & \multicolumn{1}{c}{ Gibbs Construction } \\
\hline$\left(\boldsymbol{K}_{\boldsymbol{v}}, \boldsymbol{B}_{\text {eff }}\right)$ & \multicolumn{1}{c}{$\in\left[\tilde{\boldsymbol{\Lambda}}_{\min }, \tilde{\boldsymbol{\Lambda}}_{\max }\right]$} \\
\hline$(4,65)$ & {$[663,690]$} & {$[496,509]$} \\
$(4,70)$ & {$[663,690]$} & {$[606,626]$} \\
$(17.5,95)$ & {$[663,690]$} & {$[619,647]$} \\
$(17,80)$ & {$[663,690]$} & {$[382,398]$} \\
\hline
\end{tabular}

Table 2. Table of characteristic parameters associated with a given hybrid equation of state model. The parameter pairings in the leftmost column denote the vBag parameters for the quark model that the DBHF model transitions into. For reference, the purely nuclear DBHF model yields values of $\left(M_{\max }, R_{\max }, \Lambda_{1.4}\right)=\left(2.298 M_{\odot}, 12.47,603\right)$. The maximum mass values are in units of $M_{\odot}$, and the radii values are in units of $\mathrm{km}$, and $\Lambda_{1.4}$ is dimensionless.

\begin{tabular}{ccccccc}
\hline & \multicolumn{3}{c}{ Maxwell Construction } & \multicolumn{3}{c}{ Gibbs Construction } \\
\hline$\left(\boldsymbol{K}_{\boldsymbol{v}}, \boldsymbol{B}_{\text {eff }}\right)$ & $\boldsymbol{M}_{\max }$ & $\boldsymbol{R}_{\max }$ & $\boldsymbol{\Lambda}_{\mathbf{1 . 4}}$ & $\boldsymbol{M}_{\max }$ & $\boldsymbol{R}_{\max }$ & $\boldsymbol{\Lambda}_{\mathbf{1 . 4}}$ \\
\hline$(4,65)$ & 2.120 & 12.470 & 603 & 2.141 & 12.120 & 449 \\
$(4,70)$ & 2.156 & 12.470 & 603 & 2.145 & 12.260 & 544 \\
$(17.5,95)$ & 2.231 & 12.470 & 603 & 2.145 & 12.220 & 570 \\
$(17,80)$ & 2.118 & 12.470 & 603 & 2.135 & 11.830 & 345 \\
\hline
\end{tabular}

The tidal relations in Figure 3 illustrate that many of the features discussed for the mass-radii relations translate over to the curves relating the stellar mass to the tidal deformability. While a brief deviation occurs near values at the top of the curves produced by the hybrid models, star configurations below $2 M_{\odot}$ are indistinguishable from one another. This visualization corroborates the data from the two tables above, and provides further insight into why the Maxwell-constructed models fail the $\Lambda_{1.4}$ constraint. As seen in the description of Table 2, the nuclear DBHF model fails both the $M_{\max }$ and $\Lambda_{1.4}$ constraints, and, with the Maxwell-constructed phase transitions occurring at relatively higher masses, it becomes very apparent as to why these models fail the $\Lambda_{1.4}$ constraint. While the $\left(K_{v}, B_{\text {eff }}\right)=(4,70),(17.5,95)$, and $(17,80)$ models provide the necessary conditions to adhere to the maximum mass constraint because the radii for these high mass configurations are very similar, the compactness $\mathcal{C}$ values are still very similar to that of the nuclear model.

All of the data presented in this study illustrate how the hybrid models under Gibbs construction produce softer-more compact-configurations, and, as seen by Equation (13), the compactness is a driving force in the $\Lambda$ computation. The presence of mixed quark-hadron phases resulting from these 
types of phase transitions soften the equation of state in such a way that these hybrid models are able to adhere to all of the constraints provided by the multi-messenger observations.
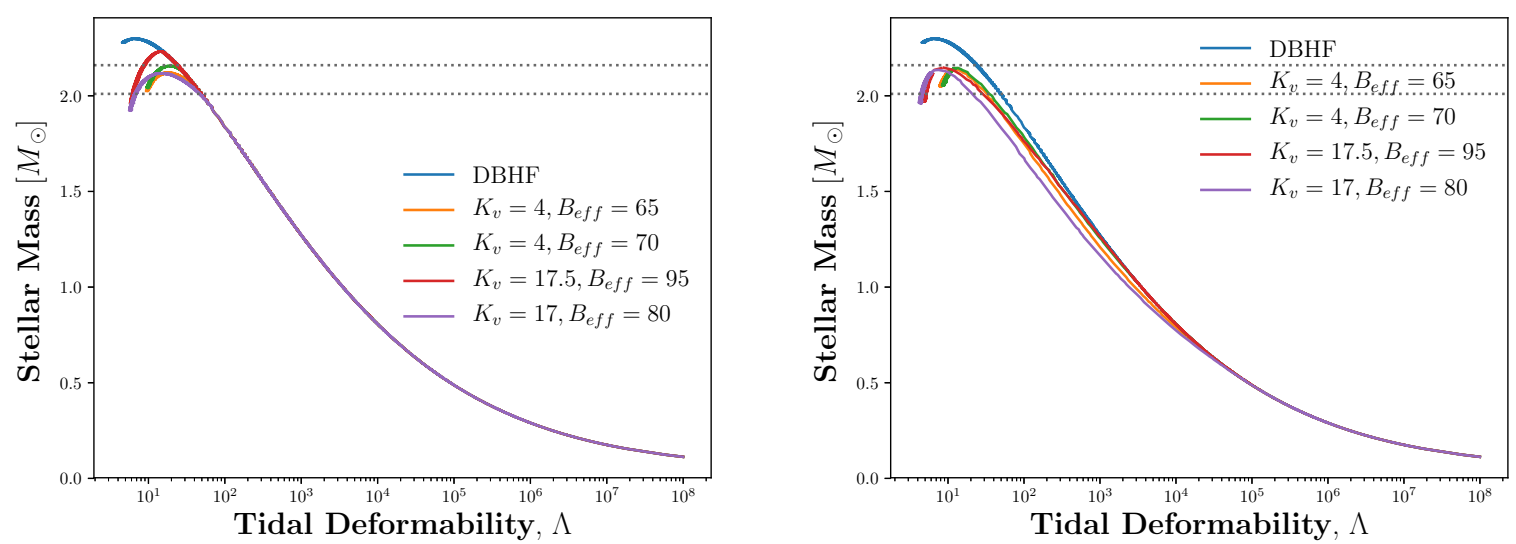

Figure 3. The Mass-Deformability curves for a component star obtained by solving Equations (5)-(13) for the various hybrid configurations. The image to the left displays the tidal behavior of Maxwell constructed hybrid models, while the one to the right does the same for Gibbs constructed models.

Lastly, the stiffness illustrated by the quark matter branches for all the hybrid models makes it difficult to delineate them from one another. Even with the deviations from the nuclear models at higher masses, especially for the Maxwell-constructed models, the tidal parameters can at times be almost indistinguishable from that of the purely nuclear model. For the Gibbs models used in this study, it is a bit difficult to understand which flavor composition is favored as the $\left(K_{v}, B_{\text {eff }}\right)=(4,65)(2 f)$ and $\left(K_{v}, B_{\text {eff }}\right)=(17,80)(3 \mathrm{f})$ models have tidal parameter values that are closer to the favorable nominal values, while the $\left(K_{v}, B_{\text {eff }}\right)=(4,70)(2 \mathrm{f})$ and $\left(K_{v}, B_{\text {eff }}\right)=(17.5,95)$ (3f) models produce values that resemble the nuclear and Maxwell-constructed models.

\section{Conclusions}

In conclusion, by our analysis with the vBAG model, the masquerade and flavor camouflage problem are not resolved by constraints on the tidal deformability of coalescing binary neutron stars. While it is still possible to detect certain hybrid models [8-10], we have shown that-given a particular parameter configuration-it can be difficult to determine the nuances of a given model using $\Lambda$. Specifically focusing on the values provided by GW170817, the mass values $M_{1}$ and $M_{2}$ lie in regions of the curve that make it nearly impossible to delineate from one another-let alone the quark contributions in the inner core. For higher mass configurations, without the aide of high-precision data, it still difficult to distinguish between EoS models due to the close proximity to one another. Additionally, properly extracting and estimating the parameters of an individual star-like its mass and radius-from gravitational radiation is extremely difficult. While techniques continue to rapidly improve, current parameter estimation methods for the NS observables do not help resolve the masquerade or flavor camouflage problems.

Although relativistic tidal deformations did not resolve these issues, other dynamical signatures my serve as a remedy. Signatures producing g-mode oscillations may serve as a better tool to decipher the properties of hybrid models [38]. The type of behavior that is quantified by $\Lambda$ is related to the non-radial f-mode oscillations at the surface of the star. G-mode oscillations develop deeper in the star's interior, making it a better indicator for behavior in regimes occupied by quark matter. 
Author Contributions: B.I. performed the tidal polarizability calculations and M.S. performed the calculations for results from the TOV equation. T.K. provided the DBHF equation of state and guided the overall study. P.J. wrote parts of the manuscript and provided intellectual input. W.W. provided the numerical code for studying phase transitions

Funding: P.J. is supported by a grant from the U. S. National Science Foundation PHY-1608959. W.W. is supported by the Natural Science Foundation of China under Grant No. 11547021 and the China Scholarship Council.

Conflicts of Interest: The authors declare no conflict of interest.

\section{References}

1. Glendenning, N.K. Compact Stars: Nuclear Physics, Particle Physics and General Relativity, 2nd ed.; Springer: New York, NY, USA, 2000; p. 468.

2. Baym, G.; Hatsuda, T.; Kojo, T.; Powell, P.; Song, Y.; Takatsuka, T. From hadrons to quarks in neutron stars: A review. Rep. Prog. Phys. 2018, 81. [CrossRef]

3. Alford, M.; Braby, M.; Paris, M.; Reddy, S. Hybrid Stars That Masquerade as Neutron Stars. Astrophys. J. 2005, 629, 969-978. [CrossRef]

4. Wei, W.; Irving, B.; Klähn, T.; Jaikumar, P. Camouflage of the phase transition to quark matter in neutron stars. arXiv 2018, arXiv:1811.09441.

5. Abbott, B.P.; Abbott, R.; Abbott, T.D.; Acernese, F.; Ackley, K.; Adams, C.; Adams, T.; Addesso, P.; Adhikari, R.X.; Adya, V.B.; et al. GW170817: Observation of Gravitational Waves from a Binary Neutron Star Inspiral. Phys. Rev. Lett. 2017, 119. [CrossRef]

6. Goldstein, A.; Veres, P.; Burns, E.; Briggs, M.S.; Hamburg, R.; Kocevski, D.; Wilson-Hodge, C.A.; Preece, R.D.; Poolakkil, S.; Roberts, O.J.; et al. An Ordinary Short Gamma-Ray Burst with Extraordinary Implications: Fermi-GBM Detection of GRB 170817A. Astrophys. J. Lett. 2017, 848, L14. [CrossRef]

7. Wang, H.; Zhang, F.W.; Wang, Y.Z.; Shen, Z.Q.; Liang, Y.F.; Li, X.; Liao, N.H.; Jin, Z.P.; Yuan, Q.; Zou, Y.C.; et al. The GW170817/GRB 170817A/AT 2017gfo Association: Some Implications for Physics and Astrophysics. Astrophys. J. Lett. 2017, 851, L18. [CrossRef]

8. Christian, J.E.; Zacchi, A.; Schaffner-Bielich, J. Signals in the tidal deformability for phase transitions in compact stars with constraints from GW170817. Phys. Rev. D 2019, 99, 023009. [CrossRef]

9. Paschalidis, V.; Yagi, K.; Alvarez-Castillo, D.; Blaschke, D.B.; Sedrakian, A. Implications from GW170817 and I-Love-Q relations for relativistic hybrid stars. Phys. Rev. D 2018, 97, 084038. [CrossRef]

10. Nandi, R.; Char, P. Hybrid Stars in the Light of GW170817. Astrophys. J. 2018, 857, 12. [CrossRef]

11. Hinderer, T. Tidal Love Numbers of Neutron Stars. Astrophys. J. 2008, 677, 1216-1220. [CrossRef]

12. Flanagan, E.; Hinderer, T. Constraining neutron-star tidal Love numbers with gravitational-wave detectors. Phys. Rev. D 2008, 77, 021502. [CrossRef]

13. Damour, T.; Nagar, A. Relativistic tidal properties of neutron stars. Phys. Rev. D-Part. Fields Gravit. Cosmol. 2009, 80, 084035. [CrossRef]

14. Binnington, T.; Poisson, E. Relativistic theory of tidal Love numbers. Phys. Rev. D 2009, 80, 084018. [CrossRef]

15. Bini, D.; Damour, T.; Faye, G. Effective action approach to higher-order relativistic tidal interactions in binary systems and their effective one body description. Phys. Rev. D 2012, 85, 124034. [CrossRef]

16. Gralla, S.E. On the ambiguity in relativistic tidal deformability. Class. Quantum Gravity 2018, 35, 085002. [CrossRef]

17. Postnikov, S.; Prakash, M.; Lattimer, J.M. Tidal Love numbers of neutron and self-bound quark stars. Phys. Rev. D 2010, 82, 024016. [CrossRef]

18. Landry, P.; Poisson, E. Relativistic theory of surficial Love numbers. Phys. Rev. D 2014, 89, 124011. [CrossRef]

19. Abbott, B.; Abbott, R.; Adhikari, R.; Ageev, A.; Allen, B.; Amin, R.; Anderson, S.B.; Anderson, W.G.; Araya, M.; Armandula, H.; et al. Analysis of LIGO data for gravitational waves from binary neutron stars. Phys. Rev. D 2004, 69, 122001. [CrossRef] 
20. Hinderer, T.; Lackey, B.D.; Lang, R.N.; Read, J.S. Tidal deformability of neutron stars with realistic equations of state and their gravitational wave signatures in binary inspiral. Phys. Rev. D-Part. Fields Gravit. Cosmol. 2010, 81, 123016. [CrossRef]

21. Maggiore, M. Gravitational Waves: Volume 2: Astrophysics and Cosmology, 1st ed.; Oxford University Press: Oxford, UK, 2018; p. 752.

22. Tolman, R.C. Static Solutions of Einstein's Field Equations for Spheres of Fluid. Phys. Rev. 1939, 55, 364-373. [CrossRef]

23. Oppenheimer, J.; Volkoff, G. On Massive Neutron Cores. Phys. Rev. 1939, 55, 374-381. [CrossRef]

24. Love, A. The yielding of the earth to disturbing forces. R. Soc. Publ. 1909, 82, 73-88. [CrossRef]

25. Van Dalen, E.N.E.; Fuchs, C.; Faessler, A. Effective Nucleon Masses in Symmetric and Asymmetric Nuclear Matter. Phys. Rev. Lett. 2005, 95, 022302. [CrossRef] [PubMed]

26. Van Dalen, E.N.E.; Fuchs, C.; Faessler, A. The relativistic Dirac-Brueckner approach to asymmetric nuclear matter. Nucl. Phys. A 2004, 744, 227-248. [CrossRef]

27. Gross-Boelting, T.; Fuchs, C.; Faessler, A. Covariant representations of the relativistic Brueckner T-matrix and the nuclear matter problem. Technical report. Nucl. Phys. A 1999, 648, 105-137. [CrossRef]

28. Akmal, A.; Pandharipande, V.R.; Ravenhall, D.G. Equation of state of nucleon matter and neutron star structure. Phys. Rev. C 1998, 58, 1804-1828. [CrossRef]

29. Fuchs, C.; Wolter, H.H. Modelization of the EOS. Eur. Phys. J. A 2006, 30, 5-21. [CrossRef]

30. Klähn, T.; Blaschke, D.; Typel, S.; Van Dalen, E.N.E.; Faessler, A.; Fuchs, C.; Gaitanos, T.; Grigorian, H.; Ho, A.; Kolomeitsev, E.E.; et al. Constraints on the high-density nuclear equation of state from the phenomenology of compact stars and heavy-ion collisions. Phys. Rev. C-Nucl. Phys. 2006, 74, 35802. [CrossRef]

31. Glendenning, N.K. First-order phase transitions with more than one conserved charge: Consequences for neutron stars. Phys. Rev. D 1992, 46, 15. [CrossRef]

32. Klähn, T.; Fischer, T. Vector Interaction Enhanced Bag Model for Astrophysical Applications. Astrophys. J. 2015, 810, 134. [CrossRef]

33. Abbott, B.P.; Abbott, R.; Abbott, T.D.; Acernese, F.; Ackley, K.; Adams, C.; Adams, T.; Addesso, P.; Adhikari, R.X.; Adya, V.B.; et al. Properties of the Binary Neutron Star Merger GW170817. Phys. Rev. X 2019, 9, 011001. [CrossRef]

34. Binary, R.; Antoniadis, J.; Freire, P.C.C.; Wex, N.; Tauris, T.M.; Lynch, R.S.; Van Kerkwijk, M.H.; Kramer, M.; Bassa, C.; Dhillon, V.S.; et al. A Massive Pulsar in a Compact. Sci. New Ser. 2013, 340, 448. [CrossRef]

35. Rezzolla, L.; Most, E.R.; Weih, L.R. Using Gravitational-wave Observations and Quasi-universal Relations to Constrain the Maximum Mass of Neutron Stars. Astrophys. J. Lett. 2018, 852, L25. [CrossRef]

36. Radice, D.; Perego, A.; Zappa, F.; Bernuzzi, S. GW170817: Joint Constraint on the Neutron Star Equation of State from Multimessenger Observations. Astrophys. J. Lett. 2018, 852, L29. [CrossRef]

37. Radice, D.; Dai, L. A Multimessenger parameter estimation of GW170817. Eur. Phys. J. A 2019, 55. [CrossRef]

38. Wei, W.; Barry, M.; Klähn, T.; Jaikumar, P. Lifting the veil on quark matter in compact stars with core g-mode oscillations. arXiv 2018, arXiv:1811.11377.

(C) 2019 by the authors. Licensee MDPI, Basel, Switzerland. This article is an open access article distributed under the terms and conditions of the Creative Commons Attribution (CC BY) license (http:/ / creativecommons.org/licenses/by/4.0/). 